Fernando Ferreira Carneiro ${ }^{1}$

Maria da Graça Hoefel ${ }^{1}$

Marina Aparecida Malheiros Silva ${ }^{2}$

Alcebíades Renato Nepomuceno ${ }^{3}$

Cleidiane Vilela ${ }^{4}$

Fernanda Rocha Amaral ${ }^{4}$

Graciele Pollyanna M. Carvalho ${ }^{4}$

Jaqueline Leite Batista ${ }^{4}$

Patrícia Abreu Lopes ${ }^{4}$

\section{Mapeamento de vulnerabilidades socioambientais e de contextos de promoção da saúde ambiental na comunidade rural do Lamarão, Distrito Federal, 2011}

\author{
Mapping socio-environmental vulnerability and environmental \\ health promotion in a rural community of Lamarão, Federal \\ District, Brazil, 2011
}

${ }^{1}$ Professores Adjuntos do Departamento de Saúde Coletiva, Faculdade de Ciências da Saúde e Núcleo de Estudos de Saúde Pública (CEAM/UnB). Brasília, DF, Brasil.

${ }^{2}$ Médica do Programa Saúde da Família da Secretaria de Estado de Saúde do Distrito Federal (SES/DF), Comunidade do Lamarão, Brasília, DF, Brasil.

${ }^{3}$ Acadêmico do Curso de Farmácia, Faculdade de Ciências da Saúde, Universidade de Brasília (UnB), Brasília, DF, Brasil.

${ }^{4}$ Acadêmicas do Curso de Enfermagem, Faculdade de Ciências da Saúde, Universidade de Brasília (UnB), Brasília, DF, Brasil.

\section{Contato:}

Fernando Ferreira Carneiro

Universidade de Brasília. Faculdade de Ciências da Saúde. Departamento de Saúde Coletiva. Campus Universitário Darcy Ribeiro, s/n - Asa Norte. Brasília-DF.

CEP: 70910-900.

Email:

fernandofc@unb.br

Este trabalho não foi subvencionado.

Recebido: 15/02/2011

Revisado: 07/05/2012

Aprovado: $17 / 05 / 2012$

\section{Resumo}

Objetivo: realizar atividade de ensino e extensão com alunos de graduação para construção de diagnóstico participativo sobre as condições de vida, ambiente e trabalho em núcleo populacional rural, visando subsidiar ações de promoção da saúde pela equipe de agentes comunitários da Estratégia Saúde da Família. Métodos: trabalho realizado no Núcleo Rural do Lamarão, no Distrito Federal, de outubro/2010 a janeiro/2011. Alunos, conjuntamente com agentes comunitários de saúde, por meio de entrevistas, visitas e oficinas, aplicaram instrumentos de Estimativa Participativa Rápida e de construção de mapa de vulnerabilidade socioambiental e de contextos de promoção da saúde ambiental. Resultados: identificaram-se como promotores da vida na comunidade: diversidade de produção de alimentos, liberdade e segurança, união e organização da comunidade, geração de empregos e natureza. Foram considerados ameaçadores à vida: cultura alimentar, uso inadequado de agrotóxicos, uso incorreto de equipamentos de proteção individual, pulverização aérea, falta de lazer, falta de transporte, uso de drogas lícitas e ilícitas. Conclusão: a partir do diagnóstico, será construído um projeto de intervenção participativo que incorporará características de ensino, pesquisa e extensão na relação Saúde, Trabalho, Ambiente e Nutrição.

Palavras-chaves: saúde rural; saúde ambiental; saúde do trabalhador; saúde da família; promoção da saúde.

\begin{abstract}
Objective: To involve undergraduate students in an extension activity aimed at conducting a participatory diagnosis on life, environment, and work conditions in a rural settlement, with the purpose of raising goals for future health promotion actions performed by community health agents of the Family Health Strategy. Methods: The study was held between October 2010 and January 2011 in the rural community of Lamarão, located in the Federal District in Brazil. By means of interviews, visits, and workshops, students and community health agents used Participatory Rapid Appraisal instruments as well as tools for mapping socio-environmental vulnerability and health promotion related issues. Results: The following were identified as life promotion factors in the community: food production diversity, freedom and safety, unity and community organization, job generation, and nature. Considered as threatening to life were: food culture, improper use of pesticides, inadequate use of personal protective equipment, aerial spraying, lack of leisure, lack of transportation, use of licit and illicit drugs. Conclusion: A participatory intervention project will be developed, which will incorporate teaching, research and extension activities regarding Health, Work, Environment, and Nutrition.
\end{abstract}

Keywords: rural health; environmental health; occupational health; family health; health promotion. 


\section{Introdução}

Os estudos sobre as condições de saúde da população do campo apontam para um perfil de saúde mais precário da população rural se comparada à urbana. No campo, ainda existem importantes limitações de acesso e qualidade dos serviços de saúde, bem como uma situação deficiente de saneamento ambiental. O processo de "modernização conservadora" ${ }^{5}$ da agricultura no Brasil ainda tem agravado mais esse quadro, na medida em que criou novos riscos socioambientais para a saúde dessa população (ALESSI; NAVARRO, 1997; CARNEIRO et al., 2007).

O fenômeno da urbanização acelerada, associada ao aumento populacional, já vinha se expressando no cenário brasileiro desde o início da segunda metade do século passado, notadamente a partir dos anos 19601970. Naquele momento o Brasil passou a viver o chamado "milagre econômico", que no campo significou um avanço das relações capitalistas, com intenso processo de modificação das relações e do processo de trabalho, associado a um forte componente repressivo aos direitos dos cidadãos e aos movimentos sociais. Passou a ocorrer então um processo de intensa mecanização, utilização de agroquímicos (dentre os quais se incluem os agrotóxicos), diminuição do emprego da força de trabalho, expansão da fronteira agrícola, ênfase na monocultura, desmatamentos, queimadas, danos ambientais intensos e descontrolados (PINHEIRO et al., 2009).

Toda esta discussão sugere que, no Brasil, existe um quadro de franco desfavorecimento da população rural em relação à urbana, no que se refere às condições de vida, trabalho e saúde. Alguns autores questionam a visão de que o "rural" seja uma esfera atrasada, arcaica, passiva e superada, mas sim de que é necessário o estabelecimento de políticas públicas justas e inadiáveis que resgatem esta imensa dívida social, cultural, ambiental e sanitária com as populações do campo (PINHEIRO et al., 2009).

Um dos grandes desafios para o Sistema Único de saúde (SUS) é garantir de forma adequada o direito a saúde para as populações do campo. A Estratégia Saúde da Família (ESF) apresenta-se como uma importante política para contribuir nesse processo.

A Política Nacional de Atenção Básica (BRASIL, 2011) caracteriza a ESF como um conjunto de ações no âmbito individual e coletivo, que abrangem promoção e proteção da saúde, prevenção de agravos, diagnóstico, tratamento, reabilitação e manutenção da saúde.

O objetivo do trabalho foi promover uma atividade acadêmica com alunos de graduação para a construção de um diagnóstico participativo sobre as condições de vida, ambiente e trabalho e para subsidiar a equipe da ESF em futuras ações de promoção à saúde junto a essa população.

\section{Métodos}

O presente trabalho foi realizado como atividade da disciplina de graduação "Ambiente, Saúde e Trabalho" ligada ao Departamento de Saúde Coletiva da Faculdade de Ciências da Saúde da Universidade de Brasília (UnB). Trata-se de um diagnóstico participativo realizado entre os meses de outubro de 2010 a janeiro de 2011, no Núcleo Rural Lamarão, com a participação de acadêmicos, docentes, equipe de saúde e comunidade da região. O trabalho foi submetido e dispensado de análise pelo Comitê de Ética em Pesquisa com Seres Humanos da Faculdade de Ciências da Saúde da UnB, em 08/05/2012.

O Núcleo Rural Lamarão, está localizado a 70 km do centro de Brasília, onde atua uma equipe da ESF do centro de saúde número 08 da Região Administrativa do Paranoá. A comunidade se encontra na região do Programa de Assentamento Dirigido do Distrito Federal (PAD/DF), programa concebido e implantado pelo Governo Distrital no início de 1977 com o objetivo de ocupar grandes extensões do cerrado com o agronegócio moderno, oferecendo terras a produtores da região sul do país. Dentre as diversas comunidades do PAD/ DF encontra-se a Colônia Agrícola Lamarão, que possui uma população de aproximadamente 332 habitantes distribuídas em 28 propriedades (GHESTI, 2011).

Para construir um diagnóstico participativo das questões de Saúde, Ambiente e Trabalho juntamente com a comunidade do Lamarão-DF, foram utilizadas algumas abordagens e instrumentos, como a Estimativa Rápida Participativa - ERP (DI VILLAROSA, 1993), e foi elaborado o mapa de vulnerabilidade socioambiental e de contextos de promoção da saúde ambiental (CARNEIRO; VIANA; PESSOA, 2010). Esta última atividade foi desenvolvida pelos discentes e docentes da disciplina de Ambiente, Saúde e Trabalho, da UnB, na forma de uma oficina de um dia em parceria com a Equipe de Saúde da Família da região e com a participação de representantes da comunidade que acolheram o convite.

A ERP se apoia em três princípios: a) coleta de dados pertinentes e necessários; b) coleta de informações que reflitam condições locais e situações específicas; c) envolvimento da comunidade na definição de seus próprios problemas e na busca de soluções, o que contribui para maior aceitação das intervenções posteriores (DI VILLAROSA, 1993).

\footnotetext{
${ }^{5}$ Segundo Delgado (2001), a "modernização conservadora" da agricultura brasileira significou a elevação do nível de investimentos de capital no campo, mas manteve ou até concentrou ainda mais a propriedade da terra no Brasil. Esta modernização também levou à precarização das relações de trabalho, além da elevação dos riscos socioambientais vinculados às atividades desse setor.
} 
Dessa forma, a ERP foi aplicada utilizando-se de três técnicas: a observação de campo da região do Lamarão-DF, entrevistas com informantes-chave e visitas domiciliares $^{6}$. Essa abordagem qualitativa visa identificar os problemas e fatores de risco por meio de entrevistas semiestruturadas com atores sociais, e por fotografias e visitas in loco para reconhecer o território, dimensionar os problemas sanitários e, assim, delinear o diagnóstico de campo da região.

Os informantes para a ERP foram selecionados pelo Agente Comunitário de Saúde (ACS) que vive na comunidade, priorizando as pessoas que viviam há muito tempo no local. O mesmo agente, com o auxílio de toda a equipe de ESF, selecionou os convidados para a oficina de mapeamento, com base na diversidade de faixas etárias e ocupações. As entrevistas tiveram como foco as características sociodemográficas e históricas do grupo. As fotografias foram realizadas pela equipe de estudo visando registrar os momentos mais importantes do trabalho.

Nesse trabalho também foi aplicada e aprimorada a metodologia de mapeamento de vulnerabilidades socioambientais e de contextos de promoção da saúde ambiental (BÚRIGO et al., 2009), onde participaram 20 pessoas representando a comunidade e a equipe da ESF. Os participantes consistiam, em sua maioria, em trabalhadores, moradores, lideranças comunitárias, professores e ACS (4 agentes).

Segundo Goldstein e Barcellos (2008), os métodos de mapeamento podem ser utilizados como instrumento didático e de debate com a população leiga sobre suas condições socioeconômicas e a inserção em seu território. Esses autores ressaltam que os mapas devem ser pensados e produzidos a partir de um processo educativo de ambas as partes (pesquisadores e população envolvida), na busca de um melhor conhecimento sobre o território, os determinantes e condicionantes ambientais e sociais e sua influência no desenvolvimento dos agravos de saúde da população. As técnicas participativas para definir a percepção geográfica de espaço servem para compartilhar os conhecimentos gerados de maneira conjunta sobre cada região, permitindo agregar novas informações que muitas vezes não estão presentes nas bases de dados oficiais.

Os participantes relacionaram em uma matriz o que ameaçava e o que promovia a vida em seu território de forma a registrar suas percepções e observações acerca dos processos ali vividos. Foi elencada uma diversidade de fatores que impactam de forma positiva e negativa a vida. Essa matriz foi inspirada nos estudos de Breilh (2003) sobre monitoramento estratégico e participativo, pois permite captar aspectos de vulnerabilidade à vida, ao mesmo tempo em que aponta as potencialidades existentes no território.
Para facilitar a apresentação dessas percepções, a comunidade desenhou um mapa representando a dinâmica da vida comunitária, caracterizando os principais fatores presentes na matriz.

\section{Construção da agenda, estratégias e instrumentos de trabalho}

Após a escolha do campo de atuação, definiu-se a agenda de trabalho do grupo juntamente com equipe de saúde rural. A equipe foi dividida em três grupos para desenvolver as seguintes atividades: observação do local entrevistas com informantes-chave e visita domiciliar. Os três grupos realizaram todas as atividades pelo menos uma vez. Acordou-se também um dia na agenda para que fosse realizada a construção do mapa de vulnerabilidade socioambiental e de contextos de promoção da saúde ambiental juntamente com os membros da comunidade na forma de uma oficina, onde o mesmo foi construído em grupo.

\section{Resultados e discussão}

\section{Observação do território}

Durante os dias de observação do ambiente, o modo de trabalho e a maneira que essas vertentes influenciam na saúde da população da comunidade do Lamarão, verificou-se que se trata de uma região composta por 28 chácaras e uma agrovila, onde residem famílias que foram distribuídas no território de acordo com o PAD/ $\mathrm{DF}$, ocupando uma região com solo fértil para a produção de alimentos, tendo as hortaliças como os produtos de maior destaque.

Na agrovila encontra-se um centro comunitário que funciona como unidade de saúde, igrejas, um campo de futebol, além de alguns comércios como bares, sorveteria e oficina. Quanto ao trabalho na lavoura, encontrou-se desde pequenas chácaras com produção de hortaliças até grandes propriedades, que comercializam produtos orgânicos em toda região do DF.

As pequenas propriedades utilizam como mão de obra a própria família, que realiza desde o preparo da terra até a venda dos produtos colhidos. Utilizam agrotóxicos na plantação, aplicados com bombas costais, muitas vezes sem o uso de EPIs. Os médios proprietários utilizam equipamento veicular, muitas vezes alugado, para a aplicação. As grandes propriedades do agronegócio circundam a comunidade e utilizam a pulverização aérea de agrotóxicos. Na mesma região existe uma fazenda, com grande produção de alimentos orgânicos, sem o uso de agrotóxicos.

A hidrografia da região é formada pelos córregos Lamarão, Cariru, São Bernardo, Pindaibal, Rio Jardim,

${ }^{6}$ As entrevistas foram realizadas pelos discentes da UnB acompanhados pelos ACS e a enfermeira da ESF. 
além de outros. A bacia hidrográfica que abastece a região do Lamarão é a do Baixo Jardim, que tem como seu principal curso de água o córrego Lamarão. Durante a observação de campo na nascente que abastece a agrovila, verificou-se que os 50 metros de reserva obrigatória não eram respeitados. A cerca estava danificada, o pasto estava muito próximo e o gado possuía acesso a área.

\section{Informações levantadas com informantes-chave}

A comunidade do Lamarão contou, no seu início, com apenas vinte famílias. Hoje esse número chega a cento e vinte famílias. A região surgiu a partir da desapropriação de grandes propriedades de famílias goianas que praticavam agricultura de forma extensiva. Em função do PAD/DF na década de 1970, foram realocados para a comunidade do Lamarão em pequenas propriedades de até 7 hectares. Em seus lugares foram assentadas famílias gaúchas com experiência na produção de cereais, que obtiveram apoio financeiro e de tecnologias modernas de produção.

A comunidade do Lamarão, hoje com quinze anos, possui chacareiros que detêm a concessão de direito relativo à propriedade, sendo responsável pelas benfeitorias nelas realizadas. A região atualmente conta com uma população bastante heterogênea, formada principalmente por baianos e mineiros, atraídos pelas possibilidades de trabalho no campo.

No início de sua formação, a comunidade passou por várias dificuldades, entre elas destacam-se a educação precária, a falta de transporte e a pobreza. Embora a maior parte do desenvolvimento tecnológico tenha sido destinada às áreas de grande produção agrícola, a comunidade tem acompanhado também o grande desenvolvimento da região representado principalmente pela chegada de luz elétrica, asfaltamento das principais vias da comunidade e diminuição da pobreza dos moradores.

Todas as famílias possuem casas de alvenaria, quase não existindo na região casas com piso em chão batido. Grande parte dos trabalhadores do campo possui uma renda de até dois salários mínimos, além das bonificações obtidas com a boa produção. Outros ainda contam com auxílios como o Bolsa Família e o Pão e Leite, ambos programas do governo. Os moradores comentam que não existem pobres na região e não falta trabalho.

Por outro lado, este desenvolvimento vem associado à alta rotatividade de trabalhadores, o que tem levado a um aumento de roubos e consumo de drogas na região, como observado por Moreira (2002).

Diante desses aspectos, a comunidade tem tido cada vez mais necessidade de uma representação política, que tem sido feita por meio da associação dos moradores, juntamente com os representantes de igrejas e os ACS, embora a Empresa de Assistência Técnica e Extensão Rural (Emater) também tenha a sua importância reconhecida. Os ACS relataram que o posto de saúde tem programas preconizados pelo Ministério da Saúde para atender a comunidade. Estes programas parecem ter grande aceitação por parte dos moradores. O principal deles trabalha com grupos de hipertensos, visto que outras doenças são pouco frequentes no Lamarão segundo o PSF local. Os agentes comentaram que as pessoas vivem até idades avançadas. Os casos graves são encaminhados para as cidades mais próximas, Planaltina ou Paranoá.

Um ponto negativo observado na região trata-se dos meios de transporte, ainda bem precários, com ônibus para as cidades mais próximas só duas vezes ao dia.

\section{Visita domiciliar}

Outra atividade que auxiliou no diagnóstico situacional da comunidade foi o acompanhamento de algumas visitas domiciliares realizadas por ACS. Durante a visita, o agente atualiza o formulário de cadastro familiar do PSF, identifica as relações familiares, conhece o contexto de vida da família e as condições de habitação, o que possibilita a realização das ações de promoção e prevenção da saúde direcionadas à realidade vivenciada. Por meio do acompanhamento das visitas, foi possível perceber a relação que se estabelece entre a equipe de saúde e a comunidade. O vínculo, o comprometimento e a confiança depositada na equipe de saúde foram alguns aspectos observados nesse momento.

\section{Construção do mapa de vulnerabilidades socioambien- tais e de contextos de promoção da saúde ambiental}

Após o grupo ter realizado um levantamento de informações a respeito da comunidade e suas relações, partiu-se para a construção do mapa de vulnerabilidade socioambiental e de contextos de promoção da saúde ambiental. Para isto, reuniu-se a equipe de saúde rural, os alunos, os professores e alguns membros chaves da comunidade (lideranças idosas e também jovens). Foi solicitado aos moradores que relatassem, segundo a opinião de cada um, os fatores que promovem e os que ameaçam a vida na comunidade. Os fatores apontados foram registrados em duas tabelas. Os alunos e professores serviram como facilitadores do processo, animando a discussão. O resultado apresentado no Quadro 1 retrata o que foi discutido e priorizado conforme registrado.

O quadro de priorização construído pela comunidade a partir dos fatores que ameaçam e promovem a vida, representa uma demonstração clara da "inteligência popular" no processo de reconhecimento de sua realidade como abordado por Breilh, (2003, p. 943). Os elementos destacados no Quadro 1 coincidem com os achados das entrevistas com informantes-chave, das visitas domiciliares e da observação do território. 
Quadro 1 Fatores que ameaçam e promovem a vida segundo membros da comunidade rural do Lamarão, DF, 2011

\begin{tabular}{|l|l|}
\hline O que promove a vida na comunidade & \multicolumn{1}{|c|}{ O que ameaça a vida na comunidade } \\
\hline & \\
\hline & - Cultura alimentar \\
- Diversidade de produção de alimentos & - Mal uso de agrotóxicos \\
- Liberdade e segurança & - Uso incorreto de EPl's \\
- União e organização da comunidade & - Pulverização aérea \\
- Disponibilidade de emprego & - Falta de lazer \\
- Natureza & - Falta de transporte \\
& - Uso de drogas lícitas e ilícitas \\
\hline
\end{tabular}

É interessante observar a importância dada à alimentação. O tema apareceu como primeiro item tanto da coluna de promoção da vida, quanto da coluna de ameaça. O paradoxo existente na comunidade é que apesar da diversidade de produção de hortaliças existe um baixo consumo das mesmas. Isto pode estar relacionado à cultura alimentar de boa parte dos trabalhadores que vieram de outras regiões como a nordeste, segundo relato de um dos membros da equipe de saúde da família.

A partir desse quadro/matriz construiu-se o mapa onde os participantes da oficina desenharam esses fatores assim como as características do território comunitário

\section{Considerações finais}

O diagnóstico participativo realizado com o auxílio dos docentes e acadêmicos da disciplina Ambiente, Saúde e Trabalho e com a participação da equipe da ESF como facilitadores dessa construção deu voz à comunidade, que percebeu a possibilidade de analisar a sua condição de vida. Essa percepção inicial pode ser a ferramenta chave para o planejamento das ações de mudança e promoção da saúde.
A estratégia desenvolvida, em função de sua simplicidade e de sua capacidade de criar pontes de diálogo com a comunidade, mostrou-se uma ferramenta importante para ser aproveitada pela Atenção Básica para atuar frente a questões de Saúde, Ambiente e Trabalho.

As atividades realizadas na comunidade do Lamarão-DF pelos docentes e acadêmicos, como a observação do território, as entrevistas com informantes-chave, as visitas domiciliares, foram essenciais para a construção do vínculo com a equipe da ESF da região, a comunidade e a UnB, possibilitando a realização do início de um trabalho interdisciplinar e participativo.

A proposta da disciplina é a continuidade dessa construção no decorrer dos semestres seguintes com a utilização dos resultados encontrados para viabilizar projetos de ensino, pesquisa e extensão que possam trazer benefícios de aprendizado aos acadêmicos das áreas da saúde, aos docentes, à equipe da ESF e, principalmente, à comunidade rural na busca pela saúde, levando em conta as formas de trabalho e o ambiente em que vivem. Os projetos em construção estão com foco na problemática da segurança alimentar, do meio ambiente e de agrotóxicos, com o compromisso de fortalecer a vigilância tanto nutricional, quanto de saúde ambiental.

\section{Contribuições de autoria}

Carneiro, F. F.: concebeu o projeto, apoiou com bibliografias, no levantamento de campo e na análise; coordenou a elaboração do manuscrito e sua revisão crítica; participou da aprovação da versão final. Hoefel, M. da G.: concebeu o projeto, apoiou com bibliografias, no levantamento de campo e na análise; participou da elaboração do manuscrito e na sua revisão crítica; aprovou a versão final. Silva, M. A. M.: concebeu o projeto, apoiou no levantamento de dados; participou da elaboração do manuscrito e na sua revisão crítica; aprovou a versão final. Nepomuceno, A. R.; Vilela, C.; Amaral, F. R.; Carvalho, G. P. M.; Batista, J. L.; Lopes, P. A.: apoiaram no levantamento de dados e na análise; contribuíram na elaboração do manuscrito e na sua revisão crítica; aprovaram a versão final. 


\section{Agradecimentos}

O presente trabalho tornou-se possível graças à parceria da Equipe de Saúde da Família da região, ao apoio da Universidade de Brasília nos trabalhos de campo e à receptividade da comunidade rural do Lamarão. Também agradecemos à Pesquisadora do NESP-UnB Lara Queiroz Viana Braga pelo apoio na preparação da equipe e durante os trabalhos de campo.

\section{Referências}

ALESSI, N. P.; NAVARRO, V. L. Saúde e trabalho rural: o caso dos trabalhadores da cultura canavieira na região de Ribeirão Preto, São Paulo, Brasil. Cadernos de Saúde Pública, Rio de Janeiro, v. 13, supl. 2, 1997. Disponível em: <http://www.scielosp.org/scielo. php?pid=S0102-311X1997000600010\&script $=$ sci arttext\&tlng $=\mathrm{es}>$. Acesso em: 1 jan. 2011.

BRASIL. Ministério da Saúde. Secretaria de Atenção a Saúde. Política Nacional de Atenção Básica. Disponível em: < http://www.saude.gov.br>. Acesso em: 18 maio 2011.

BREILH, J. De la vigilancia convencional al monitoreo participativo. Ciência \& Saúde Coletiva, São Paulo, v. 8, n. 4, 2003. Disponível em: <http://www.scielo. br/scielo.php?script = sci_arttext\&pid $=$ S1413$81232003000400016 \& \operatorname{lng}=\mathrm{en} \& \mathrm{nrm}=\mathrm{iso}>$. Acesso em: 15 jan. 2011.

BÚRIGO, A. C. et al. Relato da experiência do mapeamento de riscos socioambientais e de promoção da saúde ambiental com estudantes do MST. Tempus. Actas em Saúde Coletiva, v. 3, n. 4, p. 144-148, 2009.

CARNEIRO, F. F. et al. A saúde das populações do campo: das políticas oficiais às contribuições do Movimento dos Trabalhadores Rurais Sem Terra (MST). Cadernos de Saúde Coletiva, Rio de Janeiro, v. 15, n. 2, p. 209-230, 2007.

CARNEIRO, F. F.; VIANA, L.; PESSOA, V. M. Mapeamento de vulnerabilidades socioambientais e contextos de promoção da saúde ambiental em comunidades rurais do Vale do Jaguaribe, Ceará. In: CONGRESSO LATINOAMERICANO DE SOCIOLOGIA
RURAL, AMÉRICA LATINA: REALINEAMIENTOS POLÍTICOS Y PROYECTOS EN DISPUTA, 8., Porto de Galinhas, PE, 2010. Anais... Porto de Galinhas, PE: Associação Latino-Americana de Sociologia Rural, 2010.

DELGADO, G. C. Expansão e modernização do setor agropecuário no pós-guerra: um estudo da reflexão agrária. Estudos Avançados, v. 15, n. 43, p. 157-172, 2001.

DI VILLAROSA, F. N. A estimativa rápida e a divisão do território no distrito sanitário - manual de instruções. Brasília: OPS Representação do Brasil; 1993. (Série Desenvolvimento de Serviços de Saúde, 11)

GHESTI, L. V. Programa de assentamento dirigido do Distrito Federal - PAD/DF: uma realidade que superou o sonho. Brasília. 2009. Disponível em: <http://www. coopadf.com.br/padf.php>. Acesso em: 22 jan. 2011.

GOLDSTEIN, R. A.; BARCELLOS, C.

Geoprocessamento e participação social: ferramentas para a vigilância ambiental em saúde. In: MIRANDA, A. C. et al. (Org.). Território, ambiente e saúde. Rio de Janeiro: Fiocruz, 2008. p. 205-215.

MOREIRA, E. R. F. Trabalho, ambiente e saúde: um estudo da relação entre processos produtivos, recursos hídricos e risco à saúde. Cadernos do Logepa, João Pessoa, v. 1, n. 2, p. 47-58, jul./dez. 2002.

PINHEIRO, T. M. G. et al. Saúde no campo. In: CONFERÊNCIA NACIONAL DE SAÚDE AMBIENTAL, 1., Brasília, 2009. Caderno de Textos. Brasília: Abrasco Livros, 2009. p. 25-29. 